\title{
Effect of Smoking on Maximum Voluntary Ventilation
}

\author{
Dr.Gurunath Birajdar ${ }^{1}$, Dr.Amar Barawade ${ }^{2}$, Dr.Meera Nagavekar ${ }^{3}$ \\ ${ }^{1}$ Assistant Professor,Department of Physiology, B K L WalawalkarRural Medical College, \\ Hospital and Research centre, Sawarde, Chiplun, Maharashtra. \\ ${ }^{2}$ Assistant Professor,Department of Physiology, B K L Walawalkar Rural Medical College, \\ Hospital and Research centre, Sawarde, Chiplun, Maharashtra. \\ ${ }^{3}$ Professor and Head,Department of Physiology, B K L WalawalkarRural Medical College, \\ Hospital and Research centre, Sawarde,Chiplun, Maharashtra.
}

\begin{abstract}
Cigarettes kill an estimated 5 million people annually worldwide.The world health organization reported that tobacco smoking killed 100 million people worldwide in the $20^{\text {th }}$ century. Tobacco smoke contains 4000 chemicals out of which 60 are known carcinogens which can lead to lung cancer.Smoking is the most important risk factor for increased lung function decline in adults and the rate of decline is proportionate to amount of cigarette smoked.40 healthy adult males aged between 20-50 years smoker for more than one year at Pimpri-Chinchwad area in Pune were selected .They constitute the study group (cases).40healthy adult males non-smokers aged 20-50 years were selected as control. Our study group falls in Light smokers category (smoking index1-100).Maximum voluntary ventilation was carried out using RMS Helios 401 spirometer, Chandigarh.The present study shows decreased MVV in smokers as compared to non-smokers and the difference is statistically significant .MVV involves both inspiratory and expiratory phase of ventilation and it provides overall assessment of neuromuscular co- ordination as well as elastic and flow resistive properties of the respiratory system. $M V V$ can also be viewed as measure of respiratory muscle strength.
\end{abstract}

Keywords: Smokers, Non-smokers, MVV, RMS Helios spirometer

\section{Introduction}

Cigarettes kill an estimated 5 million people annually worldwide. ${ }^{1}$ The world health organization reported that tobacco smoking killed 100 million people worldwide in the $20^{\text {th }}$ century. ${ }^{2} \mathrm{By}$ the early 2030 , tobacco related death would increase to about 100 million a year ${ }^{3 .}$ Tobacco smoking rates have decreased in industrialized countries since 1975, but there has been corresponding 50\% increase in smoking rates in low income countries. ${ }^{4}$

Tobacco smoke contains 4000 chemicals out of which 60 are known carcinogens which can lead to lung cancer. The known chemical constituents of tobacco smoke include acetone, ammonia, butane, cadmium, carbon monoxide, hydrogen cyanide, methane, toluene, naphthalene and vinyl chloride. The smoke of cigarette is acidic ( $\mathrm{pH} \mathrm{5.3)} \mathrm{and} \mathrm{nicotine} \mathrm{is} \mathrm{relatively} \mathrm{ionized} \mathrm{and} \mathrm{insoluble} \mathrm{in} \mathrm{the} \mathrm{lipids.} \mathrm{Only} \mathrm{a} \mathrm{desired} \mathrm{amount} \mathrm{of} \mathrm{nicotine}$ is absorbed if it is taken into the lungs where there is enormous surface area for lower lipid solubility. Cigarette smokers therefore, have high rate of death due to lung cancer. ${ }^{5}$

Smoking is the most important risk factor for increased lung function decline in adults and the rate of decline is proportionate to amount of cigarette smoked. ${ }^{6-7}$ Besides the direct consequences of smoking on smokers, passive smoking by non- smokers who are exposed to tobacco smoke also has shown an increased risk of respiratory and cardiovascular problems in children ${ }^{8}$

Amongst the various tests for flows and volumes, maximum voluntary ventilation is a parameter that reflects lung volume changes, respiratory muscle functioning, compliance of the thorax lung complex and airway resistance. The MVV was formerly called the maximum breathing capacity(MBC) is the largest volume of gas that can be moved in and out of the lungs in one minute by voluntary effort.The normal MVV is 125-170 litre/min. ${ }^{9}$ MVV, one of the components of Pulmonary Function Testing (PFT) has multiple uses. MVV can be as a tool for assessment of respiratory muscle weakness which occurs mostly in smokers. The purpose of this study was to compare the MVV between smokers and non-smokers.

\section{Methods}

Institute ethical committee clearance was obtained before start of the study. The present study was conducted in the Department of Physiology, Dr. D.Y. Patil medical college,Pimpri , Pune. 40 healthy adult males aged between 20-50 years smoker for more than one year at Pimpri-Chinchwad area in Pune were selected .They constitute the study group (cases).40healthy adult males non-smokers aged 20-50 years were selected as control group. 
Anthropometrical measurements were taken along with preliminary clinical examination to exclude any systemic disorder affecting respiratory and cardiovascular system.Purpose designed questionnaire were used. Maximum Voluntary Ventilation of these two groups were compared.

The Smoking Index ${ }^{10}$ : This is a parameter which is used to express the smoking exposure quantitatively. This is especially useful in defining the risk ratio of a smoking related disease. Here, the smoking index was calculated by multiplying the average number of cigarettes which was smoked per day and the duration of smoking in years. The number of cigarettes meant, the average number of cigarettes which was smoked per day in the past seven days.

According to the smoking index, the smokers can be classified into:

1. Light smokers (Smoking index1-100)

2. Moderate smokers (Smoking index101-200)

3. Heavy smokers (Smoking index $>201$ )

Our study group falls in Light smoker's category (smoking index1-100)

\section{Exclusion criteria for study group:}

1. Subjects with cardiac arrhythmias, hypertension , diabetes mellitus, ischemic heart disease., neuropathy \& any other chronic disease.

3. History of respiratory and other cardiovascular diseases.

4. Obese and underweight subjects were excluded

Maximum voluntary ventilation was carried out using RMS Helios 401 spirometer with built in computer program, was entered into the compute, using standard laboratory methods. The questionnaires were filled up and the relevant data, name, age sex, height, weight ,occupation, smoker or non-smoker, lab temperature was entered to the computer. All subjects were made familiar with the instrument and procedure for performing Maximum voluntary ventilation. Maximum voluntary ventilationwere done on the subject comfortably seated in upright position. The subject was connected to mouthpiece and was asked to breath in order to familiarize himself with equipment. During the tests subject was adequately encouraged to perform at their optimum level and also a nose clip was applied during the entire manoeuvre.

After giving rest of ten minutes, the test to obtain maximum voluntary ventilation (MVV) was carried out. The subject were instructed to breath as deep and rapid as possible through the mouthpiece for 15 seconds. Breathing should be as constant as possible. The built in mechanism of calculation in the Helios 401 spirometer gave MVV in litres per minutes. Three consecutive readings were taken for both manoeuvre by allowing rest for 10 minutes between each effort best reading was selected and noted.

MVV (Maximum voluntary ventilation): volume of air exhaled during maximum breathing efforts within a specified time period. Every test was repeated at least 3 times and the best matching result were considered for analysis. All parameters were measured by the machine. All data were expressed as Mean (S.D.). Unpaired t-test was used to assess the effect of smoking on Maximum voluntary ventilation. P-value $<0.05$ was accepted as statistically significant.

\section{Results}

In the present study 40 smokers of age range 20-50 years were compared with 40 healthy non-smokers of same age and BMI matched. The parameter studied were shown in Table $1 \& 2$.

Table 1: Age, Body mass index of smokers and non-smokers

\begin{tabular}{|l|l|l|l|}
\hline Parameters & (Non-smokers)Control & (Smokers)Cases & P Value \\
\hline & Mean (S.D.) & Mean (S.D.) & \\
\hline Age (in years) & $30.23(6.59)$ & $30.9(8.40)$ & 0.69 \\
\hline BMI ( in kg/m2) & $24.04(3.20)$ & $23.58(3.36)$ & 0.53 \\
\hline
\end{tabular}

Table 2: Comparison of Maximum voluntary ventilation between smokers and non-smokers

\begin{tabular}{|l|l|l|l|}
\hline PFT Parameters & (Non-smokers)Control & (Smokers)Cases & P Value \\
\hline & Mean(S.D.) & Mean(S.D.) & \\
\hline MVV(L/MIN) & $111.25(24.01)$ & $87.98(27.09)$ & $0^{* *}$ \\
\hline
\end{tabular}

$\mathrm{p}$ value $<0.05$ is statistically significant.

$\mathrm{p}$ value $<0.001$ is statistically highly significant.

MVV is statistically significant as indicated by $\mathrm{p}$-value. 


\section{Discussion}

There was no significant difference in the mean physical parameters like age, height, weight and body mass index on calculating the mean and the standard deviation in the smokers and non-smokers.[Table1]. Spirometry is a frequently performed lung function test, is an important tool in medical surveillance examinations of pulmonary diseases. The interpretation of lung function relies on the comparison to reference values derived from a healthy population. Mean age of non-smokers found to be 30.23 with standard deviation 6.59 Mean ( S.D.) $=30.23(6.59)$ and in smokers found to be 30.9 with standard deviation 8.40 Mean(S.D.) $=30.9(8.40)$.

The BMI (body mass index) was found to be in non-smokers Mean (S.D.) $=24.04(3.20)$ in smokers mean(S. D. $)=23.58(3.36)$.

There was no significant difference between smokers and non-smokers when age and BMI was compared. This indicates that both the groups comparable.

Maximum voluntary ventilation (L/min): In non- smokers was 111.25(24.01) and in smokers 87.98(27.09). it is highly significant statistically as $p$ value $=0.00$. [Table2]

The mean MVV in the control group was higher. The difference was statistically very highly significant.

Similar findings withPadmavatiet al. ${ }^{11}$ who found a significant difference as the cigarette smokers had lower mean value than non-smokers.

Bajentril AL, Veeranna $\mathrm{N}(2003)^{12}$ studied that 2-5 years of tobacco smoking tends to a definite tendency to narrowing of both the large and small airways and significantly lowering lung function.

Chatterjee S, Nag SK et al. (1988) ${ }^{13}$ studied on 334 healthy male non-smokers and 300 healthy male smokers of the age range of 20-60 years and found that value of MVV and PEFR is significantly lower in smokers than non-smokers.

The present study also shows decreased MVV in smokers as compared to non- smokers and the difference is statistically significant .MVV involves both inspiratory and expiratory phase of ventilation and it provides overall assessment of neuromuscular co- ordination as well as elastic and flow resistive properties of the respiratory system .MVV can also be viewed as measure of respiratory muscle strength. ${ }^{14}$ Statistically significant changes observed in the present study probably suggest that respiratory muscle strength was affected by exposure to cigarette for the duration prescribed in the study.Another explanation for abnormal smoker's ventilator function is that the smoking of cigarette by humans is believed to cause oxidative stress by several mechanisms, including direct damage by radical species and the inflammatory response induced by smoking. Oxidative stress was found to cause apoptosisand lung injury. ${ }^{15,16}$

Accelerated decline of lung function in cigarette smokers results from smoke induced inflammatory process. Inflammation begins with an increased number of macrophages in the first and second generation respiratory bronchioles. ${ }^{17}$ Airway epithelial cells (AEC) are important regulators of inflammation in the airway. They have a function in host defence and play a significant role in airway inflammation by releasing no. of a potentially important mediator of airway inflammation as well as releasing other mediators and recruiting inflammatory cells. Cigarette smoke interferes with and inhibits the normal function of AEC by variety of mechanisms. Some of these include decreases in the level of exhaled NO, enhanced release of pro inflammatory cytokines, and inhibition of airway repair process. ${ }^{18}$ A positive association between pulmonary surfactant and airway diameter has been described; surfactant proteins were observed to inhibit pulmonary inflammation. Production of particular surfactant proteins is inhibited by tobacco smoking.

\section{Conclusion}

In the present study, Maximum voluntary ventilation was performed in the 40 smokers and 40 non-smokers male aged 20-50 years.

The study concludes:

MVV was significantly reduced in smokers.

MVV was significantly reduced in smokers indicating effect of smoking on elastic and flow resistive properties of the respiratory system. It also indicates reduced respiratory muscle strength.

This study indicated positive dose response relationship between smoking and pulmonary dysfunction. Smoking cessation programmes should form a part of major strategy to prevent COPD in young people.

\section{References}

[1]. Bulletin of the WHO. International journal of public health.2006;84(6):495

[2]. WHO report: Tobacco could kill one billion by 2100,Science Daily.2008;24:71.

[3]. Yach D. Partnering for better lung health: Improving Tobacco and Tuberculosis control. Int J Tuberc Lung Dis.2000;4:693-7.

[4]. Yu J J ,Shopland D R. Cigarette smoking behaviour and consumption characteristic for the Asia-Pacific region. World smoking health.1989;14:7-9. 
[5]. Sunita Nighute, Abhijit Tiwari. A study of the pulmonary function test among smokers and non-smokers in a rural area of Gujarat. J Clinical and DiagnosticResearch.2011;5(6):1151-1153.

[6]. Gupta P C. The public health impact of tobacco.Curr Sci.2001;81:475-81.

[7]. Muir T, Zegarac M. Societal costs of exposure to toxic substances: Economic and health cost of four case studies that are candidates for environmental causation.Environ Health Perspect.2001; 109(6):885-903[PUBMED].

[8]. World Tobacco Epidemic, $3^{\text {rd }}$ edition by WHO Geneva1994;6.

[9]. KariaRitesh M. comparative study of peak expiratory flow rate and maximum voluntary ventilation between smokers and nonsmokers. National journal of medical research Volume2 Issue 2 Apr-June2012.

[10]. SK Gupta. Respiratory disorders among workers in railway workshop.Ind.Journal of Tuberculosis.1995;42:161.

[11]. Padmavathy K M. Comparative study of pulmonary function variables in relation to tyupe of smoking.Indian J Physio Pharmacol2008;52:193-6.

[12]. Bajentri AL, VeerannaN.Effect of 2-5 years of tobacco smoking on ventilator function test. Indian Med.Association 2003;1017:967,108 .

[13]. S.Chaterjee, Nag SK, DevySK.Spirometric standards for Non-smokers and Smokers of India ( Eastern Region). Japanese Journal of Physiology, 38,283-298,1988.

[14]. . Kaur Ranjeet,Singh Harjinder,Bala Kiran,Kumar Dinesh,Bhat Anjali. Lung volumes in wasted and stunted children.Novel Sci Int J of Med Sci.2012;1(5):135-138.

[15]. Halliwell B, Gutteridge Jnc :free radicals in biology and medicine $4^{\text {th }}$ edition.Oxford:Oxford University Press;2007.

[16]. Banerjee S,Maity P, Mukherjee S, Sil A K, Panda k, Chatopadhyay D, and Chaterjee I B. Black tea prevents cigarette smoke induced apoptosis and lung damage. J Inflamm.(London)2007;4:3.

[17]. Walter R, Gottliebd J , and O'Conorg T. Environmental and genetic risk factors and gene -'environmental interactions in the pathogenesis of chronic obstructive lung disease," Environ Health perspect 2000;108(supp14):733-742.

[18]. Wei X. M.. ,Kim H S , Kumar R K,Heywood G J and Thomas P S .Effects of cigarette smoke on degranulation and no production by mast cells and epithelial cells.Respir.Res2005;6(1):108-114. 